\title{
Cooperative problem solving in a cooperatively breeding primate (Saguinus oedipus)
}

\author{
KATHERINE A. CRONIN, AIMEE V. KURIAN \& CHARLES T. SNOWDON \\ Department of Psychology, University of Wisconsin, Madison \\ (Received 16 October 2003; initial acceptance 5 December 2003; \\ final acceptance 19 February 2004; published online 11 November 2004; MS. number: A9729)
}

\begin{abstract}
We investigated cooperative problem solving in unrelated pairs of the cooperatively breeding cottontop tamarin, Saguinus oedipus, to assess the cognitive basis of cooperative behaviour in this species and to compare abilities with other apes and monkeys. A transparent apparatus was used that required extension of two handles at opposite ends of the apparatus for access to rewards. Resistance was applied to both handles so that two tamarins had to act simultaneously in order to receive rewards. In contrast to several previous studies of cooperation, both tamarins received rewards as a result of simultaneous pulling. The results from two experiments indicated that the cottontop tamarins (1) had a much higher success rate and efficiency of pulling than many of the other species previously studied, (2) adjusted pulling behaviour to the presence or absence of a partner, and (3) spontaneously developed sustained pulling techniques to solve the task. These findings suggest that cottontop tamarins understand the role of the partner in this cooperative task, a cognitive ability widely ascribed only to great apes. The cooperative social system of tamarins, the intuitive design of the apparatus, and the provision of rewards to both participants may explain the performance of the tamarins.
\end{abstract}

(c) 2004 The Association for the Study of Animal Behaviour. Published by Elsevier Ltd. All rights reserved.

A wide variety of cooperative behaviour has been described in animals, including nest building, territory defence, alliance formation, mutual hunting and cooperative infant care. Cooperative behaviour is assumed to evolve through kin selection, reciprocal altruism or mutualism (Hamilton 1964; Trivers 1971). But, as noted by Tomasello \& Call (1997) and Mendres \& de Waal (2000), we have little experimental evidence of the mechanisms by which cooperation develops. In addition, there is interest in knowing whether nonhuman animals have the cognitive skills to understand that their actions depend upon the actions of another individual. What appears to be cooperation may be simply an epiphenomenon of animals being mutually attracted to the same resources without an understanding of the task.

Boesch \& Boesch (1989) described four levels of cooperation ranging from engaging in similar actions on the same prey but without coordination in space and time (similarity), to synchronous action towards the same prey (synchrony), to both spatial and temporal behavioural coordination (coordination) to performing different, but

Correspondence: C. T. Snowdon, Department of Psychology, University of Wisconsin, 1202 West Johnson Street, Madison, WI 53706-1696, U.S.A. (email: snowdon@wisc.edu). complementary, actions directed towards the same prey (collaboration). Based on observations of group-hunting behaviour of chimpanzees, Pan troglodytes, in Tai Forest, Boesch \& Boesch (1989) concluded that their chimpanzees displayed a higher degree of coordination and collaboration than chimpanzees at other sites and than seen in other species. However, in the field it is difficult to know exactly how cooperation develops and what the chimpanzees comprehend about the role of their companions when they engage in cooperative hunting. Formal, experimental studies of apes and monkeys have attempted to understand how cooperation develops and whether animals understand their role and that of their partners in displays of cooperation.

Savage-Rumbaugh et al. (1978) trained two chimpanzees, Sherman and Austin, in complementary roles to obtain a single food reward: informant and operator. These chimpanzees learned both tasks and were successful regardless of role. The operator occasionally shared food with the informant. Chalmeau (1994; Chalmeau \& Gallo 1996) developed an apparatus requiring simultaneous pulling of handles $3 \mathrm{~m}$ apart to obtain a single fruit reward. Two of six chimpanzees, the dominant male and an infant female, learned to pull the handles simultaneously. Both pulled less often when no fruits were visible 
and both looked towards the partner more often when fruits were present. The adult male received the reward on $99.5 \%$ of the trials and monitored the actions of the infant much more than vice versa. Other group members appeared to avoid the dominant male. Chalmeau et al. (1997a) used the same apparatus to test two subadult male orang-utans, Pongo pygmaeus. Latency to succeed in the task decreased quickly with experience, but, as with the chimpanzees, one orang-utan did most of the monitoring and behavioural coordination, and received the reward on $92 \%$ of the trials, frequently pushing the other male to the apparatus. In these studies, at least the dominant animal appeared to understand the need and role of the partner.

Petit et al. (1992) placed food incentives for both rhesus, Macaca mulatta, and Tonkean macaques, Macaca tonkeana, under large rocks. Tonkean macaques acted together to move the rock but showed no evidence of coordination among partners or improvement with subsequent trials. Rhesus macaques were agonistic towards each other and displayed little joint action on the rock.

Devices similar to those developed by Chalmeau have been applied to capuchin monkeys (Cebus apella) with mixed results. Chalmeau et al. (1997b) trained capuchin monkeys to pull two handles to obtain food and then separated the handles so far apart that a single monkey could not pull both. Although some monkeys pulled the handle at the same time as a partner, they did not increase pulling rate when the partner was close to the handle. Chalmeau et al. concluded that success at cooperation was simply accidental due to the joint interest of both monkeys in the apparatus and reward. In a follow-up study Visalberghi et al. (2000) kept the same individuals together throughout testing and used a Plexiglas apparatus so that the mechanisms of action and food reward would be visible. Furthermore, they provided rewards for both actors. None the less, the monkeys showed no improvement with time and were as likely to pull the handle when the partner was off the platform as on it, or to pull the handle when the partner was not pulling as when the partner was pulling. Thus, despite improvements to increase motivation and to make the task more intuitive, by using a transparent apparatus that made the food and mechanism visible, these capuchins showed no understanding of cooperation.

Mendres \& de Waal (2000) also studied capuchin monkeys using a transparent apparatus with a single reward. Capuchin monkeys displayed 60\% success in obtaining food on the first trial, declining to less than $30 \%$ by the fourth trial in a session, not surprising given that only one monkey received a reward. The rates of success were higher when both monkeys could see each other than when an opaque screen separated them. Capuchins showed higher rates of glances when pulling by a second monkey was necessary to obtain a reward than when the first monkey could obtain the reward alone, and pull rate was significantly greater when another monkey was present than when absent, indicating an understanding of the role of the partner. de Waal \& Berger (2000) used the same apparatus and reported a success rate of $89 \%$ when two rewards were used compared with $39 \%$ when only one animal received a reward. Both studies reported food exchanges between the recipient and other animal, suggesting that reciprocal food exchanges might maintain the cooperative behaviour.

Visalberghi (1997) compared chimpanzees, capuchin monkeys and children who by 24-30 months perform well on similar tasks (Brownell \& Carriger 1990). All three species showed high levels of exploration and manipulation, but chimpanzees did not display the social tolerance that children and capuchins did, and capuchins did not appear to monitor their partner or influence their partner as did children and chimpanzees. In addition to task transparency, social tolerance and close monitoring of partners, we would add the need for both actors to receive rewards for their behaviour (either immediately or through exchanges) in order to sustain cooperation. To date only three studies (Visalberghi et al. 2000; de Waal \& Berger 2000; de Waal \& Davis 2003) have provided rewards for both individuals. In some of the remaining studies, not provisioning both participants may allow dominance interactions to confound potential cooperation.

Cooperatively breeding callitrichid primates display the characteristics of an egalitarian social system and a high degree of coordinated action cited by Coussi-Korbel \& Fragaszy (1995) as optimal for social learning (Snowdon 2001). Callitrichid primates display apparent social cognitive abilities not seen in other monkeys or even in some great apes. As in many other species, marmosets and tamarins share food with infants. However, golden lion tamarin, Leontopithecus rosalia, adults tend to share food that is rare, difficult to process or novel to the infant, but familiar to the adults, suggesting social transfer of information (Price \& Feistner 1993; Rapaport 1999). Common marmosets, Callithrix jacchus, displayed imitation (Bugnyar \& Huber 1997; Voelkl \& Huber 2000). Cottontop tamarins, Saguinus oedipus, learned from observation of other group members to avoid a highly preferred food that was made noxious by the addition of invisible white pepper (Snowdon \& Boe 2003). Common marmosets learned a novel foraging task by interacting with a trained demonstrator (Caldwell \& Whiten 2003). Werdenich \& Huber (2002) found that some dyads in a group of common marmosets solved a coproduction task involving a single reward, with the dominant animal more often acting as scrounger but sharing food with the subordinate producer.

The present study was designed to examine cooperative behaviour in the sense of Boesch \& Boesch (1989) that cooperation occurs when two or more individuals act together to achieve a common goal. We developed a task for cottontop tamarins where the food and the actions involved in obtaining food would be transparent to both actors and where both individuals would benefit equally through joint action. The apparatus was designed so that two tamarins would be opposite each other when acting and thus able to observe each other's actions directly. To be certain that each individual's actions were based on understanding the necessity of the actions of the other, we tested tamarins when alone with the apparatus and when with their partner. 


\section{EXPERIMENT 1}

\section{Methods}

\section{Subjects}

We tested four pairs of unrelated, but paired, cottontop tamarins at the Psychology Department at the University of Wisconsin, Madison. Each pair consisted of an adult female and a vasectomized adult male housed together. Subjects ranged in age from 4.5 to 8 years and had been paired together for at least 3 years. The tamarins were housed in indoor cages constructed of anodized aluminium framing and polyurethane-coated steel mesh. Cages measured either $160 \times 236 \times 93 \mathrm{~cm}(\mathrm{~L} \times \mathrm{H} \times \mathrm{W})$ (pairs, Go and Es; Sa and Gr; Sc and In) or $236 \times 220 \times 186 \mathrm{~cm}$ (pair $\mathrm{Ga}$ and $\mathrm{He}$ ). Cages included natural branches, wooden planks and ropes to simulate an arboreal environment. All tamarins were housed in rooms containing multiple cages, which were isolated from each other visually by opaque fabric sheets. For additional husbandry information, see Ginther et al. (2001).

\section{Apparatus}

To test the tamarins' ability to cooperate we designed a small, clear plastic box with two transparent sliding trays inside, one protruding from each side (Fig. 1). The apparatus measured $15 \times 13 \times 4 \mathrm{~cm}(\mathrm{~L} \times \mathrm{H} \times \mathrm{W})$, with ledges extending $20 \mathrm{~cm}$ on either side. The portion of the tray that was external to the apparatus contained a handle for an animal to pull on and extend the tray. The handles of the apparatus were $20 \mathrm{~cm}$ apart and positioned opposite each other so that a tamarin could only pull one tray at a time. The portion of the tray inside the apparatus had two holes located $4.5 \mathrm{~cm}$ and $9 \mathrm{~cm}$ from the edge of the handle of the tray. Rewards were placed in these holes on the top tray. When the apparatus was baited with rewards and both handles were fully extended, the holes in the trays aligned vertically, then a reward was released from each side of the apparatus. The trays of the apparatus had screws extending from them that allowed for manual resetting of the trays once extended or for immobilizing the tray. In addition, elastic bands could be attached to the screws so that the trays could be automatically retracted once tension on the handle was released. All cooperation testing utilized elastic bands to retract the handles, minimizing the presence of the experimenter.

The ledge on either side of the box was large enough for the tamarins to stand or sit on. During testing, the apparatus was hung securely from the inside of subjects' home cage door at a height of approximately $120 \mathrm{~cm}$. The experimenter manually baited the apparatus from outside the cage at the beginning of each trial. Immediately after baiting the apparatus, the experimenter left the cage area until the apparatus needed to be rebaited. Pilot tests determined which reward food best motivated each pair of subjects. Raisins were used to reward simultaneous extension of the apparatus handles by two pairs (Go and Es; $\mathrm{Ga}$ and $\mathrm{He}$ ), while the other two pairs (Sc and In; Sa and Gr) received Kix brand cereal (General Mills, Minneapolis, Minnesota, U.S.A.) as rewards.

\section{Training}

To prepare the tamarins for the experiment, we first habituated them to the apparatus. Then, through the use of shaping techniques, we taught the tamarins to manipulate the handles of the apparatus. The shaping technique required the tamarins to learn to pull the handle in incremental stages, each an extension of the previous stage. We first rewarded the tamarins for touching the apparatus in any way, then only for standing on the ledge, then only for standing on the ledge while touching the handle, then only for touching the handle while a researcher moved the handle towards them. Finally, tamarins received rewards for pulling the handle completely on their own, at which point the reward was released by the apparatus rather than provided by the experimenter. During this initial training phase, we set the apparatus so that one tray was already extended and locked in place, requiring only the other side to be pulled to release a single reward. The tray that required pulling was not attached with elastic bands, making the task as easy as possible. We alternated the side that required pulling from one training session to the next. We trained both tamarins in a pair alternately during a session, and we recorded when an animal received a reward and the action for which it was rewarded on a laptop computer. Training sessions occurred on a nearly daily basis.

When tamarins were proficient at pulling apparatus handles, we initiated a second phase of training to habituate the subjects to encountering resistance when pulling the tray handles. We added an elastic band to one tray and left the other tray in the extended, locked position. The tray with tension was alternated from session to session. Only the tray with tension was baited with a reward, and tamarins quickly were able to pull against resistance. We attempted to train each tamarin individually by occupying the second tamarin with food while the subject was at the apparatus. However, a single training session almost always included training bouts with both tamarins. In the final phase we habituated the tamarins to manipulating the apparatus at the same time, by allowing both subjects to be rewarded. The apparatus was set up with a rubber tension band on only one tray, with the other tray able to slide freely. Both sides of the apparatus were baited with rewards, and tamarins received rewards after both handles had been extended, although not necessarily simultaneously.

Before formal cooperation testing began, the following criteria had to be met: (1) both tamarins had to pull the handle with tension during a session and (2) both handles had to be pulled within $3 \mathrm{~s}$ on all trials in a training session. The median number of total training sessions before both pair members met the criteria was 26.5 sessions (range 14-32 sessions). Training session lengths depended on motivational levels, but averaged $10.5 \mathrm{~min}$ per pair. All training took place at least $1 \mathrm{~h}$ after feeding with at least $24 \mathrm{~h}$ between sessions.

\section{Testing procedure}

For the test sessions, elastic bands were attached to both trays. The only action for which tamarins were rewarded 
(a)

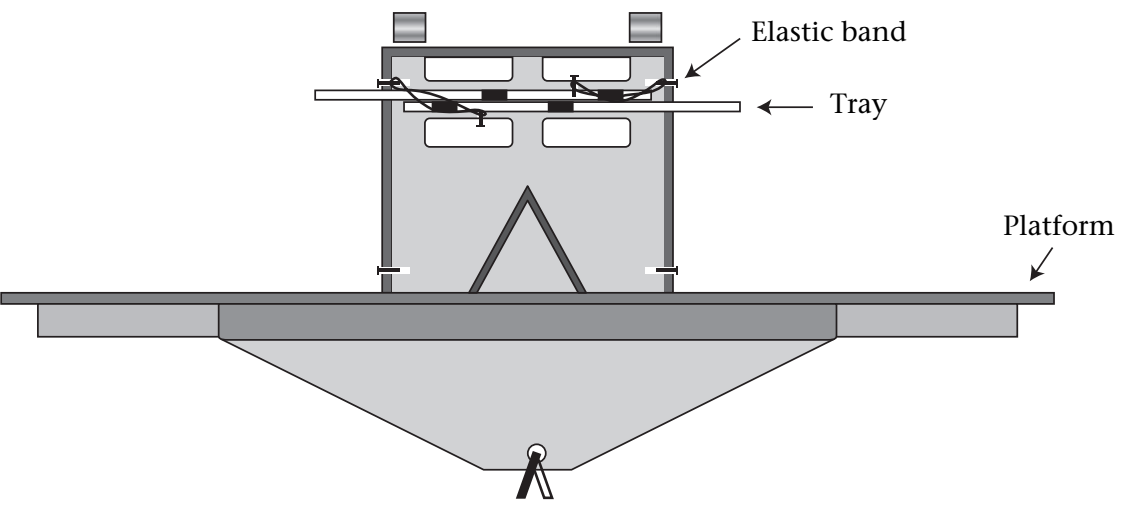

(b)

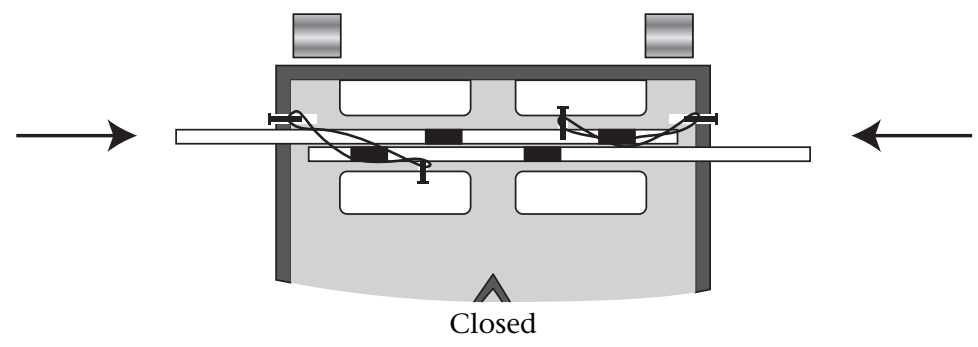

(c)

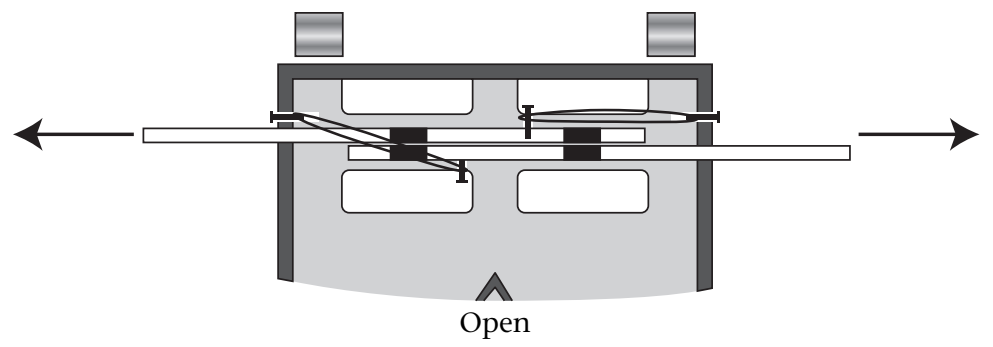

Figure 1. Diagram of apparatus. (a) Side view showing the opposing platforms on which two tamarins could sit and the two trays attached by elastic bands. The dark areas indicate holes in the tray. A food reward was placed in each hole in each top tray. Only when both trays were extended did the holes on both trays line up. The two food rewards dropped to the floor of the apparatus, one on each side of the baffle. Each animal could receive a food reward. Trays in closed (b) and open (c) positions. When the elastic bands were attached, both trays had to be pulled simultaneously in order for the food to drop.

during testing was the simultaneous full extension of both handles.

We videotaped all testing using a Sony Handycam (Sony, Tokyo, Japan) with Maxell T-120 GX-Silver VHS tapes (Maxell Corporation of America, New Jersey, U.S.A.) positioned on a tripod $90 \mathrm{~cm}$ in front of the subjects' cage door. We focused the camera to record activity in the immediate vicinity of the apparatus $(15 \mathrm{~cm}$ above and below and $20 \mathrm{~cm}$ to the left and right of the apparatus). We began a session as soon as the experimenter finished baiting the apparatus and left the area. We ended a session when tamarins had completed all trials or had ceased pulling handles for at least 3 min during a trial. In pilot testing, we found that tamarins that lost interest in the cooperation apparatus due to their inability to coordinate pulling attempts and receive rewards quickly extinguished pulling behaviour. By terminating sessions after $3 \mathrm{~min}$ had elapsed since either tamarin pulled a handle, we minimized the risk of extinguishing pulling behaviour. Each pair participated in 20 cooperation sessions. We conducted testing between 1000 and 1200 hours, with 1-5 days elapsing between tests.

Each session consisted of a maximum of eight trials. The first trial began when the apparatus was baited with the first set of rewards. A trial ended when rewards were released from the apparatus. As soon as the tamarins had finished eating the rewards, we rebaited the apparatus and began the next trial. For the two pairs receiving raisins as rewards (Go and Es; $\mathrm{Ga}$ and $\mathrm{He}$ ), approximately $20 \mathrm{~s}$ elapsed between trials. Processing time (the time it took the monkey to eat the reward) was faster on tests utilizing Kix cereal as rewards with less than $5 \mathrm{~s}$ between trials for Sc and In, and Sa and Gr.

\section{Data scoring}

We scored behaviour from the videotaped testing sessions. We recorded pulling events for each individual 
and summed this to describe the pulling behaviour of both partners in each trial. We defined a pull as a full extension of an apparatus handle, using one or both hands, until the screw of the handle encountered the apparatus wall producing an audible click. We did not score partial extensions or touching an apparatus handle as pulls. Only full extension and retraction of the handle was counted as a pull. Cases in which a tamarin fully extended a handle, let it retract, then fully extended it again without removing its hands from the apparatus were scored as two individual pulls. We also classified pulling behaviour in relation to the tamarin's partner. We scored pulls as Together when the animal's partner was present on the opposite platform of the apparatus and facing the handle. Any other situation in which an animal pulled a handle was scored as an Alone pull. Alone pulls included pulling while the partner was absent from the apparatus, pulling while the partner was present on the opposite platform but oriented away from the handle, as well as instances in which a tamarin pulled a handle and held it open until its partner approached the opposite platform and pulled the other handle.

We also recorded data to document the style of pulling used each time rewards were released from the apparatus. Tamarins used two styles of pulling during cooperation testing: Regular or Sustained. Regular pulls occurred when an animal released the handle immediately after extension. Sustained pulls were recorded when a tamarin retained tension on the apparatus handle for at least $1 \mathrm{~s}$ after extension. We scored rewards that were obtained from simultaneous pulling efforts (a regular pull by each tamarin) as Simultaneous, and those obtained when one tamarin extended a handle and held it open until its partner pulled the other handle as Sequential (at least one tamarin executed a Sustained pull). Sustained pulls are an interesting adaptation to solving the cooperation task, and we thought they would be useful in discerning subjects' understanding of the role of a partner in the cooperative task.

We also recorded data on the identity of the first animal to show pulling behaviour in each trial and latency to reward. We defined latency to reward as the elapsed time between when the experimenter finished baiting the apparatus and when the rewards were released from the apparatus.

The number of pulls executed per trial was used to calculate an efficiency measure, similar to 'per cent cooperation' for capuchin monkeys of Visalberghi et al. (2000). We defined efficiency percentage as the minimum number of pulls (per pair) required to obtain all rewards in a testing session, divided by the actual number of pulls executed (per pair) per trial multiplied by 100 . For example, if a test consisted of four cooperation trials, then eight pulls were the minimum number of pulls necessary to obtain all four sets of rewards. If the pair of subjects executed 16 pulls during this particular test, then their efficiency was 50\% (8/ $16 \times 100)$. Efficiency rates were calculated using both Together and Alone pulls. A higher efficiency rate reflects fewer extraneous pulls and more behavioural coordination between partners.

\section{Data analysis}

We used a sample size of four pairs for data analyses. We tabulated each session separately, then made comparisons between blocks of 10 sessions. We used correlated samples $t$ tests to compare performance between the two blocks of sessions. All tests were two-tailed.

\section{Results}

\section{Success rate and efficiency}

A cooperation trial was considered a success if the pair of subjects obtained rewards from their pulling behaviour regardless of the number or types of pulls involved. The mean \pm SE success rate for the four pairs of subjects was $96.84 \pm 1.01 \%$ in a combined total of 634 cooperation trials. Additional measures such as efficiency rates and types of pulls suggest that the high success rate was not due to chance alone.

One pair of tamarins, Sa and Gr, started out at a very high efficiency rate in the first block of 10 sessions $(70.5 \%)$ and maintained that level of efficiency in the second block of sessions (67.8\%). The other three pairs of tamarins increased in efficiency from the first block of sessions to the second block (first 10 sessions: $37.9 \pm 4.23 \%$; second 10 sessions: $47.5 \pm 5.87 \%$ ). However, the increase in efficiency was not significant $\left(t_{2}=2.41, P=0.137\right.$, power $\left.=0.29\right)$.

\section{Pull measures}

We compared Alone and Sustained pulls between the first and second blocks of 10 sessions to evaluate whether tamarins had an understanding of the cooperation task or whether their success in obtaining rewards was due to random action. Excluding the pair that demonstrated $70 \%$ efficiency on the first 10 sessions, the remaining three pairs performed a significantly lower percentage of Alone pulls per session in the second block of 10 sessions (block 1: $34.3 \pm 3.82 \%$; block 2: $23.8 \pm 3.38 \%$; paired $t$ test: $t_{2}=4.35, P<0.05$, power $=0.62$; Fig. 2 ). In addition, the percentage of rewards resulting from Sustained pulling increased significantly from the first block $(55.89 \pm 6.51 \%)$ to the second block $(76.83 \pm 6.89 \%$;

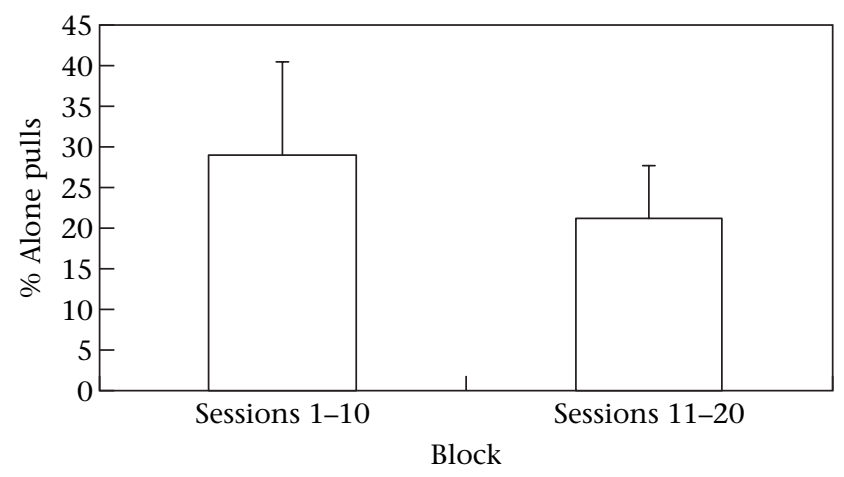

Figure 2. Percentage of Alone pulls in sessions 1-10 and sessions 1120. The percentage of Alone pulls decreased significantly between blocks of sessions. 
paired $t$ test: $t_{2}=7.94, P<0.02$, power $=0.94$; Fig. 3) also excluding the pair with high efficiency. The mean latency to receive rewards was short and did not change over the course of the experiment (first block: 16.7 s; second block: $14.3 \mathrm{~s})$.

\section{Discussion}

Cottontop tamarins spontaneously solved a cooperation task from the very first session that they were required to pull on the apparatus simultaneously. The mean success rate $(96.8 \%)$ of the cottontop tamarins we tested was much higher than some studies with brown capuchins, C. apella, another New World monkey species (Chalmeau et al. 1997b; Mendres \& de Waal 2000; Visalberghi et al. 2000) and even of great apes (Chalmeau 1994; Chalmeau \& Gallo 1996; Chalmeau et al. 1997a). Only de Waal \& Berger (2000) report comparable levels (89\%) in capuchin monkeys when two rewards were used. The motivation of both tamarins remained high throughout the testing.

The efficiency rate we calculated is closely related to the 'per cent cooperation' in Visalberghi et al's (2000) study with $C$. apella. The mean efficiency rate of all four pairs of cottontop tamarins was $46.0 \%$ in the first block of 10 sessions and $52.6 \%$ in the second block, both of which were higher than that reported by Visalberghi et al. (2000) for capuchins, C. apella (27.3\%). Mendres \& de Waal (2000) did not provide a comparable measure in their studies of capuchin monkeys.

One pair demonstrated an efficiency rate of $70 \%$ in the first block of 10 sessions and performance remained stable in the second block. The remaining three pairs showed significant decreases in the percentage of Alone pulls and significant increases in the percentage of Sustained pulls between the first and second blocks, implying an acquisition of behaviour leading to efficient cooperation.

However, these results alone are not sufficient to demonstrate cooperation as a cognitive adaptation. In the presence of a visible and highly preferred food reward, both individuals may be attracted to the apparatus and pull at a high rate with the apparent success at a cooperative task being an artefact of the attractiveness of the food reward (Visalberghi et al. 2000). To evaluate this

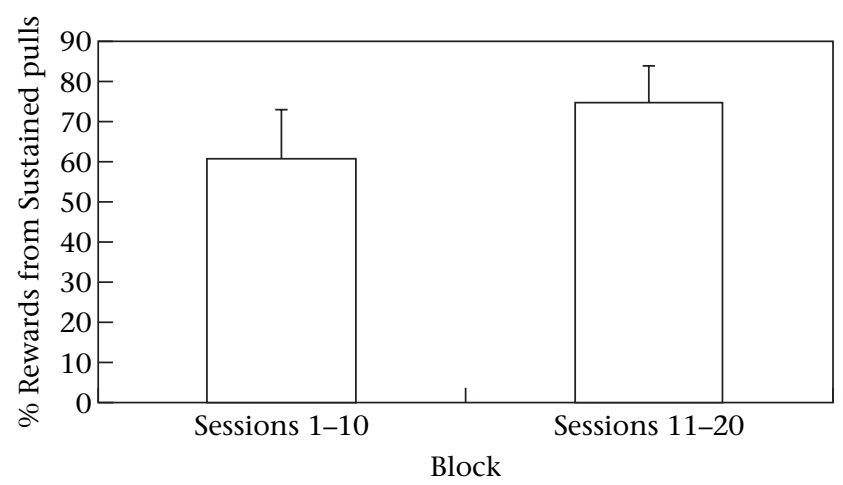

Figure 3. Percentage of rewards from Sustained pulls in sessions 1-10 and sessions 11-20. The percentage of Sustained pulls increased significantly between blocks of sessions. possibility, we need to compare the performance of individuals tested alone with the apparatus and then tested with their partner (Mendres \& de Waal 2000). If the tamarins understand the cooperation task, they should rarely pull when alone compared to when a partner is present. Experiment 2 tests this hypothesis.

\section{EXPERIMENT 2}

In this experiment, we tested tamarins in the presence of the baited apparatus alone and with the mate present to determine whether they would alter their rate of pulling in the presence or absence of a partner.

\section{Methods}

\section{Subjects}

The same four pairs of tamarins used in experiment 1 were studied in experiment 2 . Approximately 3 months elapsed between experiments, during which time the tamarins received no further testing or exposure to the apparatus. Prior to testing for experiment 2, all tamarins were acclimated to an auxiliary cage that measured $66 \times 188 \times 64 \mathrm{~cm}(\mathrm{~L} \times \mathrm{H} \times \mathrm{W})$. These auxiliary cages were always isolated visually from the home cage with opaque sheets. The auxiliary cage was connected to the home cage with $15.2-\mathrm{cm}$ diameter ventilation ducting made of thermoplastic urethane with internal wires (Hitech Hose, Inc., Chicago, Illinois, U.S.A.). We could open or close the connection to the home cage and auxiliary cage by means of a plastic door flap at each end of the connecting tube, allowing tamarins to be held in one cage or the other. The experimenter controlled door flaps from outside the cage.

Auxiliary cages are commonly used in our colony to transport animals and to add cage space for large families. All tamarins in this study had previously encountered them. Tamarins were first habituated to entering and leaving the auxiliary cage. When both tamarins appeared comfortable with this, we then habituated them to being enclosed in the auxiliary cage as a pair for increasing intervals. We assessed anxiety by monitoring long calling and chattering vocalizations, scent marking, attempts to enter/exit the auxiliary cage, and piloerection. When the pair showed little anxiety to isolation in the auxiliary cage for $3 \mathrm{~min}$, we began individual habituation. We habituated individuals to being enclosed alone in the auxiliary cage (i.e. showing no agitation while enclosed) for increasing intervals. We also monitored the anxiety of the pair member remaining in the home cage. Once both pair members remained calm throughout 3 min of separation, we began formal testing.

One pair of tamarins, Sc and In, did not progress beyond the habituation stage. During individual habituation to the auxiliary cage, the pair showed substantial anxiety to being separated, marked by vocalizations, frequent scent marking and attempts to pry open the door flap. The agitation continued for several minutes once the pair was reunited. Because these tamarins could not attend to the cooperation task after separation, they were not included 
in the formal testing. The other three pairs adjusted well to the brief separation.

\section{Apparatus}

The same cooperation apparatus used in experiment 1 was used in this experiment. The apparatus was set up in the same manner as described in experiment 1, with the exception that all subjects received raisins as rewards.

\section{Testing procedure}

As in experiment 1, all testing was videotaped using a Sony Handycam positioned on a tripod $90 \mathrm{~cm}$ in front of the tamarins' cage door, which captured action $15 \mathrm{~cm}$ above and below the apparatus, and $20 \mathrm{~cm}$ to the left and right of the apparatus. Sessions began as soon as the experimenter exited the area after baiting the apparatus and ended when subjects had completed all trials or had ceased pulling handles for at least 3 min during a trial. Each test session consisted of a Solitary portion followed by a Paired portion. Each pair received 10 test sessions. The tamarin presented with the cooperation apparatus in the Solitary portion was alternated from session to session, so each tamarin participated in five Solitary tests. Testing was conducted once daily between 1000 and 1200 hours.

During the Solitary test portion, we isolated one member of the pair in the auxiliary testing cage while its partner was presented with the cooperation apparatus for $3 \mathrm{~min}$. The apparatus was baited with two rewards at the beginning of each Solitary test portion. Since both trays needed to be fully extended for rewards to be released, the single tamarin could not be rewarded. The Paired test began when the solitary animal's partner was released from the auxiliary cage and entered the home cage. Each Paired portion contained a maximum of four trials, with $30 \mathrm{~s}$ between trials. A trial began when the apparatus was baited with two raisins and ended when the animals were rewarded for fully extending the handles at the same time. For trial 1 of the Paired test, the apparatus was already baited with the two rewards from the Solitary test.

\section{Data scoring}

All behaviour was scored from the videotaped testing sessions. A subset of $10 \%$ of sessions were scored by a second observer with an interobserver reliability of 92\% agreement for whether a pull occurred or not, 97\% agreement on whether a pull was Alone or Together and $87 \%$ agreement on whether a pull was Sustained or Regular. In experiment 2, individual pulling behaviour was recorded because individuals were tested separately. The same definitions of pulling behaviour used in experiment 1 were used to characterize pulling in this experiment: individual pulls, Together versus Alone pulls, Regular versus Sustained pulls.

We classified each pull in experiment 2 as Regular or Sustained, whereas only the pulls resulting in rewards were classified in this way during the first experiment. Timing of each pull was recorded relative to the time code of the VCR. From this, a pull rate (pulls/min) was calculated for each tamarin in each condition. We calculated an efficiency measure as in experiment 1 for each pair in the Paired portion. In addition to pulling behaviour, we recorded duration of contact with the apparatus for both tamarins in all testing sessions.

\section{Data analysis}

We conducted data analyses using a sample size of six tamarins, except for success rates and efficiency rates, which reflected the performance of the pair rather than individual tamarins.

\section{Results}

\section{Success rate and efficiency over time}

Over the 108 cooperation trials in the Paired portion of this experiment, pairs were successful on $95.3 \%$ of trials. We made comparisons between the first and second half of testing sessions (blocks of 5 sessions). Ga and He were 95\% successful in sessions 1-5 and 100\% successful in sessions 6-10; Sa and Gr performed at 100\% success in both blocks of sessions; Go and Es were only $40 \%$ successful in sessions $1-5$ but improved to $94.1 \%$ success in sessions 6-10. We calculated the efficiency rate of each pair to compare the tamarins' performance to previous studies. The mean efficiency rate in sessions $1-5$ was $30.33 \%$ and increased to $42.6 \%$ for sessions $6-10$, which was similar to results from the first experiment and higher than those previously reported in capuchin monkeys. The pair with the lowest efficiency rate in experiment 1, Go and Es, performed at very low efficiency (only 6.3\%) in sessions 1-5 but more than tripled their efficiency to $20.0 \%$ in sessions 6-10. However, Sa and Gr, the pair that performed at extraordinarily high efficiency throughout experiment 1 (69.2\%), only performed at $34.6 \%$ and $47.7 \%$ efficiency in the first and second blocks of this experiment. The third pair, $\mathrm{Ga}$ and $\mathrm{He}$, performed at approximately the same level as experiment 1, with a $50.1 \%$ and $60.1 \%$ efficiency rate in the first and second blocks, respectively.

\section{Performance in Solitary and Paired portions}

We found no significant difference in the mean percentage of time spent in contact with the apparatus in the Paired $(44.7 \pm 7.2 \%)$ versus Solitary $(30.8 \pm$ $21.0 \%$ ) portion (paired $t$ test: $t_{5}=1.72, P=0.146$, power $=0.41$ ), nor any significant change in latency to contact the apparatus in the Paired $(28.9 \pm 14.6 \mathrm{~s})$ versus Solitary $(33.4 \pm 40.6 \mathrm{~s})$ portion $t_{5}=0.37, P=0.724$, power $=0.06$ ), suggesting that the baited apparatus was attractive to tamarins regardless of partner's presence or absence. However, tamarins pulled the handles at a significantly lower rate during the Solitary portion (all sessions: $1.42 \pm 0.33$ pulls/min) than during the Paired portion (all sessions: $3.43 \pm 0.43$ pulls $/ \mathrm{min}$; sessions $1-5$ : $2.91 \pm 0.39 \mathrm{pulls} / \mathrm{min}$; sessions $6-10: 3.94 \pm 0.71$; paired $t$ tests: Solitary versus Paired: all sessions: $t_{5}=5.44$, $P<0.003$, power $=1.0$; Solitary versus Paired: sessions 1-5: $t_{5}=4.47, P<0.007$, power $=0.998$; Solitary versus Paired: sessions 6-10: $t_{5}=3.77, P<0.013$, power $=0.99$; Fig. 4). 


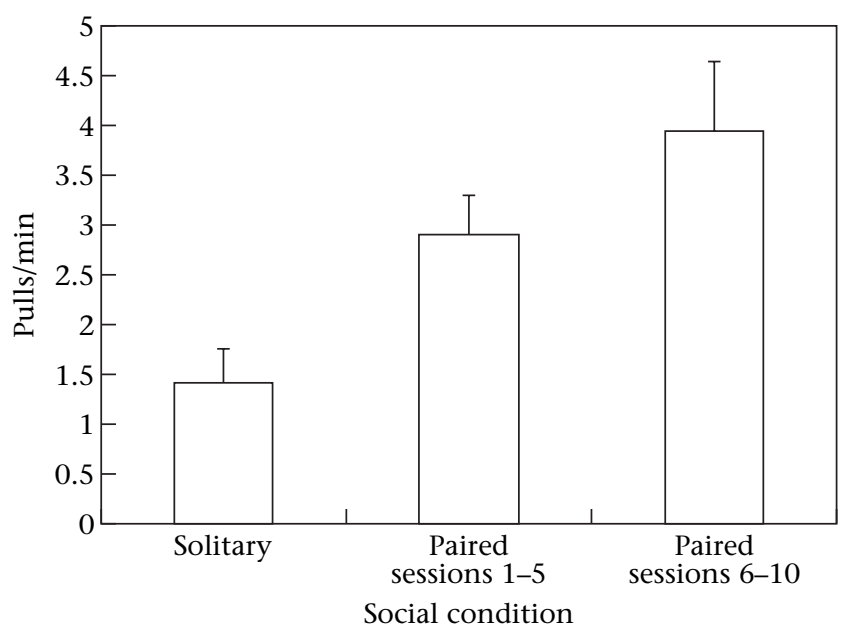

Figure 4. Number of pulls/min in experiment 2 when tamarins were solitary, and when paired in sessions $1-5$ and sessions $6-10$. Pull rate was significantly lower in the solitary condition than in the paired condition.

\section{Discussion}

Because the tamarins pulled the handles of the apparatus significantly less often when alone than when their partner was present, the results indicate that tamarins are sensitive to the need of a partner in order to successfully solve the task. There were no differences between Solitary and Paired portions in the latency to approach or time spent in contact with the apparatus, indicating that the baited apparatus was attractive to tamarins regardless of the presence or absence of the mate, and that the absence of the mate did not produce a severe behavioural disturbance that would lead to ignoring the apparatus. However, tamarins rarely pulled on the apparatus when they were alone, but pulled at significantly higher rates when the mate was present. The fact that the rates of success and efficiency were generally similar to that found in experiment 1 suggests that the experience of brief separation had little effect on the subsequent responses of the pair. Thus, as seen in the Mendres \& de Waal (2000) study of capuchin monkeys, cottontop tamarins were sensitive to the presence or absence of their partner.

\section{GENERAL DISCUSSION}

The cooperative behaviour observed in this study most likely resulted from a combination of the following factors: (1) the cooperative social system of callitrichids, (2) the intuitive structural design of the apparatus such that rewards were visible to both individuals and both individuals were able to monitor each other's behaviour, and (3) both participants received rewards when they acted together.

Cottontop tamarins have an egalitarian social system. Because nonreproductive individuals assist in infant carrying and food sharing, tamarins require a high degree of communication with one another to coordinate the transfer of infants between helpers, the alternation of roles between carrying and vigilance, and the transfer of information about novel foods to naïve young. CoussiKorbel \& Fragaszy (1995) described outcomes of social learning, two of which aid in the production of cooperative behaviour: social cohesion via synchrony and homogenization of behaviour. The authors hypothesized that the latter would emerge only in egalitarian systems. Therefore, the behavioural repertoire of the tamarins may help to explain the reason the tamarins showed a higher efficiency rate in the cooperation task than brown capuchins (Chalmeau et al. 1997b; Visalberghi et al. 2000) and great apes (Chalmeau 1994; Chalmeau \& Gallo 1996; Chalmeau et al. 1997a), species with less cooperative and less egalitarian social systems. The one exception is the de Waal \& Berger (2000) study that found similar efficiency rates when both capuchin monkeys were rewarded.

Mendres \& de Waal (2000) concluded that capuchins did not succeed in a prior cooperative study (Chalmeau et al. 1997b) because the task was not intuitive (did not allow both monkeys to see the food and the mechanism to obtain food). However, both Visalberghi et al. (2000) and Mendres \& de Waal (2000) used a task designed to be more intuitive, but they obtained conflicting results regarding the capuchins' cooperation efficiency. We also designed an apparatus inside which all mechanisms and rewards were visible to the tamarins. The tamarins were required to pull the baited tray towards themselves. The cause-and-effect relationship between the tamarins' pulling behaviour and the release of the reward was immediately clear to an observing participant. When Mendres \& de Waal (2000) eliminated visual contact between the capuchins, success rate dropped. We designed our apparatus to enable the tamarins to have an unobstructed view of their partner; each participant could monitor the other's face and actions. Other studies (Chalmeau \& Gallo 1996; Chalmeau et al. 1997a; Mendres \& de Waal 2000) reported increased rates of glancing or looking at the partner in their side-by-side cooperation tasks.

The third factor that we believe contributed to the tamarins' successful cooperation was allowing both participants to be rewarded. Few previous studies of cooperative task solving rewarded both participants. Visalberghi et al. (2000) rewarded each capuchin monkey when they simultaneously pulled the handles of their apparatus. Their capuchins frequently solved the task, but were inefficient, one animal pulling without the other present. de Waal \& Berger (2000) described two types of tasks: the 'cooperation' task provided only one reward where capuchins were successful $39 \%$ of the time in contrast to a 'mutualism' condition when both animals were rewarded and the success rate was $89 \%$, closer to the $97 \%$ success rate we found with tamarins. However, de Waal \& Berger (2000) studied capuchin monkeys housed in confined chambers with few other behavioural options. The present study and Visalberghi et al. (2000) tested animals in very large enclosures where many other activities were possible. In this regard, the high efficiency of tamarins is even more remarkable.

In Mendres \& de Waal (2000) and de Waal \& Berger (2000), food sharing was observed between the individual receiving the reward and the partner. Recent work on 
capuchin monkeys (Brosnan \& de Waal 2003) demonstrates that capuchin monkeys refuse to participate in cooperative tasks with humans if they receive a lesser reward than their companion, and they also lose interest in tests where dominants claim more than their share (de Waal \& Davis 2003). Studies on cottontop tamarins (Hauser et al. 2003) show that tamarins will share food preferentially with those who reciprocate. Werdenich \& Huber (2002) reported that only half of the pairs of common marmosets that they tested displayed cooperation. They used only a single reward, and success occurred only amongst dyads where a more dominant animal was the scrounger. However, the marmoset that scrounged food frequently shared the food with its partner. Dominance interactions may confound cooperation, but reciprocal food sharing may lead to cooperation as much as providing rewards for both individuals. Reciprocal food sharing between partners meets the requirements for cooperation as defined by Boesch \& Boesch (1989).

Cooperation studies with chimpanzees and orang-utans (Chalmeau 1994; Chalmeau \& Gallo 1996; Chalmeau et al. 1997a) show that dominant individuals understand cooperative behaviour, but the dominance interactions between the participating apes and the provision of only one reward in an experimental design do not allow evaluation of what other group members understand about cooperation.

Boesch \& Boesch (1989) described four levels of cooperation increasing in complexity: similarity, synchrony, coordination and collaboration. In our current study, the tamarins showed the second most complex form of cooperation, coordination. It is important to note that while the tamarins were being trained for this experiment, they were rewarded for performing similar actions but were never rewarded for coordinating behaviour with their partner. When the task required both partners to act together, the tamarins adjusted immediately and spontaneously.

Mendres \& de Waal (2000) observed that capuchins adjusted their pulling rate based on the presence or absence of their partner, as did the cottontop tamarins in our study. When the capuchins were solitary, they showed a pull rate of 1.2 pulls $/ \mathrm{min}$. This is similar to the solitary pull rate of $1.4 \mathrm{pulls} / \mathrm{min}$ in the cottontop tamarins. When the capuchins were tested together, the pull rate was 2.8 pulls/min, slightly less than the pull rate of cottontop tamarins when together (3.4 pulls/min). Visalberghi et al. (2000) used two rewards and an intuitive apparatus and reported only $27.3 \%$ cooperation efficiency among capuchins compared with the mean efficiency rate of $49 \%$ in the tamarins we tested.

Overall, these findings (together with those of Hauser et al. 2003) provide evidence that cottontop tamarins have developed cooperative behaviour as a cognitive adaptation. The tamarins appear to be aware of the role of their partners in cooperative actions and adjust their behaviour according to the behaviour of their partners. We expect that other species of callitrichids, given their characteristically egalitarian social system and reduced neophobia (compared with tamarins; see Day et al. 2003) would perform equally well on this intuitive task. We also expect clear demonstrations of cooperation in other species when a transparent apparatus is used, when each individual is provided with a reward for joint action (or food sharing occurs), and when social relationships between individuals involved are more egalitarian than despotic.

\section{Acknowledgments}

The research was supported by a University of Wisconsin Hilldale Student-Faculty Grant to K.A.C., and by United States Public Health Service Grant MH 29775 to C.T.S. We thank Carla Boe, Matthew W. Campbell, Kate F. Washabaugh and Sofia K. Zahed for advice and critical feedback, Andrew Mulder for constructing the apparatus and Bob Becker for the drawing of the apparatus.

\section{References}

Boesch, C. \& Boesch, H. 1989. Hunting behavior of wild chimpanzees in the Tai National Park. American Journal of Physical Anthropology, 78, 547-573.

Brosnan, S. F. \& de Waal, F. B. M. 2003. Monkeys reject unequal pay. Nature, 425, 297-299.

Brownell, C. A. \& Carriger, M. S. 1990. Changes in cooperation and self-other differentiation through the second year of life. Child Development, 61, 1164-1174.

Bugnyar, T. \& Huber, L. 1997. Push or pull: an experimental study on imitation in marmosets. Animal Behaviour, 54, 817-831.

Caldwell, C. A. \& Whiten, A. 2003. Scrounging facilitates social learning in common marmosets, Callithrix jacchus. Animal Behaviour, 65, 1085-1092.

Chalmeau, R. 1994. Do chimpanzees cooperate in a learning task? Primates, 35, 385-392.

Chalmeau, R. \& Gallo, A. 1996. What chimpanzees (Pan troglodytes) learn in a cooperative task. Primates, 37, 39-47.

Chalmeau, R., Lardeux, K., Brandibas, P. \& Gallo, A. 1997a. Cooperative problem solving by orangutans, Pongo pygmaeus. International Journal of Primatology, 18, 23-32.

Chalmeau, R., Visalberghi, E. \& Gallo, A. 1997b. Capuchin monkeys, Cebus apella, fail to understand a cooperative task. Animal Behaviour, 54, 1215-1225.

Coussi-Korbel, S. \& Fragaszy, D. M. 1995. On the relation between social dynamics and social learning. Animal Behaviour, 50, 14411453.

Day, R. L., Coe, R. L., Kendal, J. R. \& Laland, K. N. 2003. Neophilia, innovation and social learning: a study of intergeneric differences in callitrichid monkeys. Animal Behaviour, 65, 559-571.

Ginther, A. J., Ziegler, T. E. \& Snowdon, C. T. 2001. Reproductive biology of captive male cottontop tamarins as a function of social environment. Animal Behaviour, 61, 65-78.

Hamilton, W. D. 1964. The genetical evolution of social behavior. Journal of Theoretical Biology, 7, 1-52.

Hauser, M. D., Chen, M. K., Chen, F. \& Chuang, E. 2003. Give unto others: genetically unrelated cotton-top tamarin monkeys preferentially give food to those who altruistically give food back. Proceedings of the Royal Society of London, Series B, 270, 23632370.

Mendres, K. A. \& de Waal, F. B. M. 2000. Capuchins do cooperate: the advantages of an intuitive task. Animal Behaviour, 60, 523-529. 
Petit, O., Desportes, C. \& Thierry, B. 1992. Differential probability of "coproduction" in two species of macaque (Macaca tonkeana, M. mulatta). Ethology, 90, 107-120.

Price, E. C. \& Feistner, A. T. C. 1993. Food sharing in tamarins: tests of three hypotheses. American Journal of Primatology, 31, 211-221.

Rapaport, L. G. 1999. Provisioning of young in golden lion tamarins (Callitrichidae, Leontopithecus rosalia): a test of the information hypothesis. Ethology, 105, 619-636.

Savage-Rumbaugh, E. S., Rumbaugh, D. M. \& Boysen, S. T. 1978. Linguistically mediated tool use and exchange by chimpanzees. Behavioral and Brain Sciences, 4, 539-554.

Snowdon, C. T. 2001. Social processes in communication and cognition in callitrichid monkeys: a review. Animal Cognition, 4, 247-257.

Snowdon, C. T. \& Boe, C. Y. 2003. Social communication about unpalatable foods in tamarins. Journal of Comparative Psychology, 117, 142-147.

Tomasello, M. \& Call, J. 1997. Primate Cognition. New York: Oxford University Press.
Trivers, R. L. 1971. The evolution of reciprocal altruism. Quarterly Review of Biology, 46, 35-57.

Visalberghi, E. 1997. Success and understanding in cognitive tasks: a comparison between Cebus apella and Pan troglodytes. International Journal of Primatology, 18, 811-830.

Visalberghi, E., Pellegrini Quarantotti, B. \& Tranchida, F. 2000. Solving a cooperation task without taking into account the partner's behavior: the case of capuchin monkeys (Cebus apella). Journal of Comparative Psychology, 114, 297-301.

Voelkl, B. \& Huber, L. 2000. True imitation in marmosets. Animal Behaviour, 60, 195-202.

de Waal, F. B. M. \& Berger, M. L. 2000. Payment for labour in monkeys. Nature, 404, 563.

de Waal, F. B. M. \& Davis, J. M. 2003. Capuchin cognitive ecology: cooperation based on projected returns. Neuropsychologia, 41, 221-228.

Werdenich, D. \& Huber, L. 2002. Social factors determine cooperation in marmosets. Animal Behaviour, 64, 771-781. 